\title{
Community-acquired pneumonia through Enterobacteriaceae and Pseudomonas aeruginosa: diagnosis, incidence and
} predictors

\author{
H. von Baum*, T. Welte ${ }^{\#}$, R. Marre ${ }^{ø}$, N. Suttorp ${ }^{+}$and S. Ewig ${ }^{\S}$ for the CAPNETZ \\ study group
}

ABSTRACT: The aim of the present study was to determine the relevance of the presence of Enterobacteriaceae (EB) and Pseudomonas aeruginosa (PA) in patients with community-acquired pneumonia (CAP) and how the true incidence of these pathogens can be assessed.

Based on prospective data from 5,130 patients with CAP included in the German Competence Network for Community-Acquired Pneumonia (CAPNETZ), the incidence, clinical characteristics, outcome and predictors of patients with CAP due to EB and PA were studied applying strict case definitions.

The incidence of EB was 67 (1.3\%) out of 5,130, including 27 patients with bacteraemia. PA was found in $22(0.4 \%)$ out of 5,130 patients. These microorganisms were judged to be indeterminate pathogens in an additional 172 and 27 isolates, respectively. Patients with indeterminate pathogens differed considerably from those with definite isolates in terms of clinical presentation, comorbidity, pneumonia severity and outcome. Independent risk factors for EB included cardiac and cerebrovascular disease, and for PA chronic respiratory disease and enteral tube feeding. The 30-day mortality was significantly higher in patients with definite pathogens.

In the present large population, the incidence of CAP due to EB/ PA was low. The risk of the presence of these pathogens can be assessed using several predictors, which may identify those patients in need of an extended diagnostic work-up and initial antimicrobial treatment.

\section{KEYWORDS: Community-acquired pneumonia, Enterobacteriaceae, Pseudomonas aeruginosa}

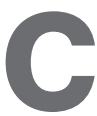
ommunity-acquired pneumonia (CAP) is characterised by three main criteria. First, it is an acute infectious injury of the lung, secondly, the pneumonia is acquired in the community, i.e. not in the hospital; and, thirdly, it occurs in a host without severe immunosuppression, i.e. not at significant risk for opportunistic pathogens. The resulting clue is that these three criteria permit a relatively confident prediction of the pathogen patterns to be expected, with Streptococcus pneumoniae being the leading pathogen, followed by atypical bacterial and viral pathogens. Thus the diagnosis of CAP and assessment of its severity represent a tough basis for decisions regarding initial treatment settings and empirical initial antimicrobial treatment [1].

Although the concept of CAP usually works well, there remain important uncertainties at its periphery. These include all pathogens which are only rarely implicated (mainly in at-risk populations), and which are associated with considerable pathogenicity but require a very different antimicrobial coverage, such as Enterobacteriaceae (EB) and Pseudomonas aeruginosa (PA). A landmark Spanish study found these pathogens in $\sim 10 \%$ of the study population [2], a finding confirmed in a recent Korean study [3]. In contrast, others found these pathogens in $\sim 1-3 \%$ [4-7].

Moreover, the concept of CAP has recently been challenged by the definition of the new pneumonia entity healthcare-associated pneumonia (HCAP) [8, 9]. The key perception behind this concept is that elderly and severely disabled patients with constant and/or recent contact with healthcare institutions, such as for dialysis,
AFFILIATIONS

*Institute for Medical Microbiology and Hygiene, Ulm University Hospital, and

•Ulm University Hospital, UIm, \# Dept of Pneumology, Hanover University, Hanover, ${ }^{+}$Dept of Infectious Diseases and Pulmonary Medicine, Charité Berlin, Berlin, and

${ }^{\S}$ Thoraxzentrum Ruhrgebiet, Dept of Respiratory and Infectious Diseases, Herne and Bochum, Germany.

\section{CORRESPONDENCE}

$\mathrm{H}$. von Baum

Institute for Medical Microbiology and Hygiene

Ulm University Hospital

Steinhövel Str. 8

D-89075 UIm

Germany

E-mail: heike.von-baum@uniklinikulm.de

Received:

June 102009

Accepted after revision:

Aug 072009

First published online:

Aug 132009 
nursing homes or hospitals, exhibit a prognostic and aetiological pattern essentially different from CAP. In particular, multiresistant pathogens, including EB and PA, are claimed to occur frequently [10-12].

The incidence, clinical presentation, prognosis and predictors of CAP due to EB/PA were studied. Moreover, patients with definite pneumonia due to $\mathrm{EB} / \mathrm{PA}$ according to strict definitions were compared with those exhibiting these pathogens but failing to meet these strict criteria in an attempt to improve understanding of what CAP due to EB/PA truly represents.

\section{MATERIALS AND METHODS Patient population}

A detailed description of the Competence Network for Community-Acquired Pneumonia (CAPNETZ) methodology is given elsewhere [13]. The present study was approved by the ethical review board of each of the participating centres, and all patients included provided informed consent.

\section{Data collection}

In the present prospective study, all demographic, clinical and diagnostic patient data were recorded using standardised Internet-based data sheets created by $2 \mathrm{mt} \circledast$ (Ulm, Germany). The study period comprised 61 months, starting on June 1, 2002 and ending on June 30, 2007, thus including four autumnwinter seasons.

\section{Microbiological processing and examination}

The methods applied have been described previously [14, 15]. In short, sputum and/or other respiratory secretions were immediately processed in the participating local microbiological laboratories according to the German Quality Standards in Clinical Microbiology and Infectious Diseases. The results of virus testing are not included in the present report.

\section{Sample processing}

All respiratory specimens and blood cultures, if available, were immediately processed in the local microbiological laboratories of the participating clinical centres. Gram-staining and culture were performed for all respiratory samples. Validated sputum, blood culture, pleural fluid, undiluted and serially diluted tracheobronchial aspirate, protected specimen brush and bronchoalveolar lavage fluid (BALF) samples were plated on sheep blood agar, Centers for Disease Control and Prevention (CDC) anaerobe 5\% sheep blood agar and chocolate, as well as Sabouraud, agar. Undiluted protected specimen brush and BALF samples were also cultured on charcoal-yeast extract agar. All Gram-negative pathogens were identified to species level according to standard methods. Standardised susceptibility testing was performed in the local microbiological laboratories. Urine was tested for the presence of S. pneumoniae and Legionella spp. antigens.

\section{Definitions}

Isolates of $\mathrm{EB} / \mathrm{PA}$ were considered definite causative pathogens when they were: 1 ) isolated from blood cultures or pleural fluid cultures; or 2) in the presence of a good-quality sputum revealing $>25$ polymorphonuclear cells and $<10$ epithelial cells per power field (total magnification $100 \times$ ) and predominant growth in culture of sputum of $\geqslant 1 \times 10^{6}$ colony-forming units (CFU) $\cdot \mathrm{mL}^{-1}$ or BALF of $\geqslant 1 \times 10^{4} \mathrm{CFU} \cdot \mathrm{mL}^{-1}$. If one of these criteria was not met, the presence of EB or PA was judged to be indeterminate.

\section{Statistical analysis}

Comparisons between groups were performed by means of the Chi-squared test for categorical variables, or Fisher's exact test in the case of small expected frequencies, and ANOVA for continuous variables including multiple comparisons. Multivariate analysis of predictive factors for 30-day mortality and CAP due to definite EB/PA was performed using binary logistic regression analysis. All analyses were performed using SPSS software (SPSS 10.0; SPSS, Inc., Chicago, IL, USA). All tests of significance were two-tailed, and $\alpha$ was set at 0.05 .

\section{RESULTS}

\section{General characteristics of study population}

Overall, 5,130 patients with CAP from 12 clinical centres throughout Germany were included in the analysis during 2002-2007. The 2,842 male and 2,288 female patients had a mean age of $60 \pm 18$ yrs. Of the patients, $65 \%(n=3,334)$ were hospitalised when first contacted for participation in CAPNETZ, and 307 (6\%) were nursing home residents. Comorbid conditions were present in 3,299 (64\%) patients, and $31 \%$ were smokers. Severity scores, as assessed using the confusion, respiratory frequency and blood pressure in those aged $\geqslant 65$ yrs (CRB-65) index, were available for $90 \%$ of the patients $(0: 37 \% ; 1: 38 \% ; 2: 13 \% ; 3: 2 \%$; and $4: 0.3 \%)$. Of the patients, $126(2.5 \%)$ required mechanical ventilation and 326 $(6.4 \%)$ died within 30 days after diagnosis. The clinical characteristics of patients without respiratory samples and no definite EB/PA were significantly different and reflected greater age, greater comorbidity, more frequent nursing home residency, greater pneumonia severity on presentation and increased mortality in the patient group with EB/PA as definite pathogens. The demographic and clinical data of patients with and without EB/PA are given in tables 1 and 2, respectively.

\section{General microbial patterns}

S. pneumoniae was identified as the predominant respiratory pathogen in the study population, followed by Mycoplasma pneumoniae. In $<5 \%$ of the patients Legionella spp., Haemophilus influenzae and Staphylococcus aureus were identified. Even less frequent were Moraxella catarrhalis and Chlamydophila pneumoniae.

\section{Diagnosis and epidemiology of EB and PA}

Respiratory samples were available from 2,102 (41\%) of the patients. In 40 patients, EB were considered the definite causative pathogen according to the predefined criteria. The most frequent $(48 \%)$ enterobacterial pathogen isolated was Escherichia coli. In contrast, EB were classified as indeterminate in an additional 172 patients. Overall 172 (81\%) out of 212 isolates yielding EB were indeterminate rather than definite pathogens.

Of the patients, 27 (40\% of all EB pneumonia cases) had definite CAP due to EB with bacteraemia through E. coli (18 cases) and Proteus mirabilis and Klebsiella spp. (three cases each), as well as Enterobacter spp., Serratia marcescens and Morganella morganii (one case each) (online supplementary material). 
TABLE 1 Demographic and clinical data of patients with and without Enterobacteriaceae (EB)

\begin{tabular}{|c|c|c|c|c|c|}
\hline $\begin{array}{c}\text { No respiratory } \\
\text { sample }\end{array}$ & $\begin{array}{c}\text { Respiratory sample with no } \\
\text { definite/indeterminate EB/PA } \\
\text { isolates }\end{array}$ & p-value ${ }^{\#}$ & Definite EB & Indeterminate EB & p-value $\quad p$-value ${ }^{+}$ \\
\hline
\end{tabular}

\begin{tabular}{|c|c|c|c|c|c|c|c|}
\hline Subjects $\mathrm{n}$ & 3009 & 1833 & & $67^{\S}$ & 172 & & \\
\hline Age yrs & $60 \pm 19$ & $58 \pm 18$ & $<0.000$ & $73 \pm 15$ & $66 \pm 14$ & $<0.000$ & 0.002 \\
\hline Male sex & 53 & 58 & 0.002 & 61 & 69 & 0.553 & 0.276 \\
\hline Hospitalisation & 69 & 56 & $<0.000$ & 94 & 73 & $<0.000$ & 0.000 \\
\hline CRB-65 score & & & $<0.000$ & & & $<0.000$ & 0.000 \\
\hline 0 & 36 & 39 & & 13 & 24 & & \\
\hline 3 & 3 & 1 & & 9 & 2 & & \\
\hline 4 & 0.4 & 0.1 & & 0 & 1 & & \\
\hline Smoking history pack-yrs & $28 \pm 22$ & $29 \pm 23$ & 0.439 & $46 \pm 40$ & $37 \pm 31$ & $<0.000$ & 0.179 \\
\hline Dyspnoea & 71 & 74 & 0.043 & 90 & 76 & 0.005 & 0.038 \\
\hline Confusion & 10 & 4 & $<0.000$ & 22 & 6 & $<0.000$ & 0.000 \\
\hline Oxygen requirement & 44 & 38 & $<0.000$ & 85 & 51 & $<0.000$ & 0.000 \\
\hline Congestive heart failure & 19 & 14 & $<0.000$ & 46 & 25 & $<0.000$ & 0.001 \\
\hline Cerebrovascular disease & 12 & 5 & $<0.000$ & 33 & 12 & $<0.000$ & 0.000 \\
\hline $\begin{array}{l}\text { Other chronic neurological } \\
\text { disorder }\end{array}$ & 8 & 3 & $<0.000$ & 15 & 8 & $<0.000$ & 0.086 \\
\hline Diabetes mellitus & 17 & 13 & $<0.000$ & 30 & 24 & $<0.000$ & 0.338 \\
\hline Enteral tube feeding & 2.0 & 0.5 & $<0.000$ & 4.5 & 6.0 & 0.008 & 0.762 \\
\hline CRP $\mathrm{mg} \cdot \mathrm{L}^{-1}$ & 78 & 74 & 0.337 & 153 & 93 & 0.026 & 0.204 \\
\hline Leukocyte count cells $\mu \mathrm{L}^{-1}$ & 11100 & 11100 & 0.784 & 12650 & 11650 & 0.006 & 0.048 \\
\hline Change in antibiotic therapy & 27 & 24 & 0.009 & 40 & 24 & 0.002 & 0.016 \\
\hline 30-day mortality & $220(7.3)$ & $82(4.5)$ & $<0.000$ & $14(20.9)$ & $12(7.0)$ & $<0.000$ & 0.002 \\
\hline
\end{tabular}

Data are presented as mean \pm SD, \% or $n(\%)$, unless otherwise indicated; C-reactive protein (CRP) concentrations and leukocyte counts are medians. PA: Pseudomonas aeruginosa; CRB-65: confusion, respiratory frequency and blood pressure in those aged $\geqslant 65 \mathrm{yrs}$. * : respiratory sample with no definite/indeterminate EB/PA isolates versus no respiratory sample; " $:$ definite EB versus respiratory sample with no definite/indeterminate EB/PA isolates; ${ }^{+}$: indeterminate EB versus definite EB; ${ }^{\text {s. : in }}$ respiratory samples $(n=40)$ and blood cultures $(n=27)$.

In seven of the bacteraemic patients, respiratory samples were available, two of which yielded an identical bacterial species to the corresponding blood cultures.

Thus the overall incidence of CAP with definite EB was only 67 $(1.3 \%)$ out of 5,130 . It was $63(1.9 \%)$ out of 3,334 in hospitalised patients and four $(0.2 \%)$ out of 1,796 in ambulatory patients. Taking into account only patients with respiratory samples available, the overall incidence was 67 (3.2\%) out of 2,102.

Applying the same criteria, definite PA pneumonia was diagnosed in 22 patients.

PA in the respiratory tract of an additional 27 patients was considered indeterminate (table 2). In nine additional cases, respiratory samples yielded Pseudomonas spp. other than PA (not further specified $(\mathrm{n}=2), P$. fluorescens $(\mathrm{n}=5)$, and P. putida and P. stutzeri $(\mathrm{n}=1$ each)) and were consequently excluded from the analysis. Overall, 27 (55\%) out of 49 isolates yielding PA were indeterminate rather than definite pathogens. Only one case had PA bacteraemia; respiratory samples from this patient were not available.

Accordingly, the overall incidence of CAP due to PA was 22 $(0.4 \%)$ out of 5,130 . PA was absent in ambulatory patients. The incidence was $22(1.0 \%)$ out of 2,102 in the subgroup with respiratory samples available.

Copathogens were isolated from the respiratory materials of six patients with definite EB (three Legionella spp., two S. pneumoniae and one $H$. influenzae) and eight patients with definite PA pneumonia (three S. aureus, two S. pneumoniae, one Streptococcus pyogenes, one M. catarrhalis and one K. pneumoniae).

The incidences of EB and PA according to different denominators are summarised in table 3 .

\section{Clinical characteristics of CAP due to EB and PA}

Comparing the population with respiratory samples $(n=1,840)$ with that classified as having definite EA/PA, patients with EB 
TABLE 2 Demographic and clinical data of patients with and without definite Pseudomonas aeruginosa (PA)

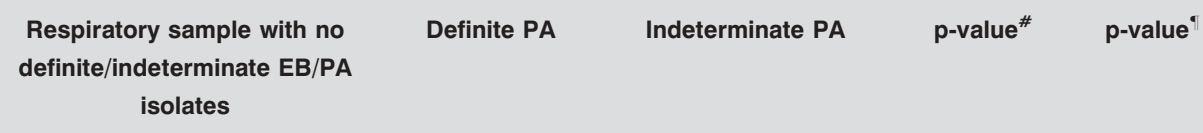

\begin{tabular}{|c|c|c|c|c|c|}
\hline Subjects $n$ & 1833 & $22^{+}$ & 27 & & \\
\hline Age yrs & $58 \pm 18$ & $64 \pm 17$ & $68 \pm 11$ & 0.101 & 0.322 \\
\hline Male sex & 58 & 59 & 70 & 0.886 & 0.406 \\
\hline Nursing home resident & 2 & 23 & 7 & $<0.000$ & 0.219 \\
\hline CRB-65 score & & & & 0.103 & 0.653 \\
\hline 0 & 39 & 32 & 19 & & \\
\hline 3 & 1 & 5 & 0 & & \\
\hline 4 & 0.1 & 0 & 4 & & \\
\hline Oxygen requirement & 38 & 82 & 59 & $<0.000$ & 0.123 \\
\hline Mechanical ventilation & 2.5 & 9 & 11 & 0.101 & 1.000 \\
\hline Chronic respiratory disease & 37 & 68 & 52 & 0.002 & 0.247 \\
\hline Diabetes mellitus & 13 & 27 & 19 & 0.039 & 0.510 \\
\hline Change in antibiotic therapy & 24 & 64 & 40 & $<0.000$ & 0.106 \\
\hline 30-day mortality & $82(4.5)$ & $4(18.2)$ & $1(3.7)$ & 0.015 & 0.160 \\
\hline
\end{tabular}

or PA were significantly older and had more comorbid conditions. Between a quarter and a fifth were nursing home residents. Severe comorbidity was reflected by more frequent enteral tube feeding. Pneumonia on presentation was more severe. All of the patients with EB bacteraemia and all with PA were hospitalised. Mortality was approximately five times higher (tables 1 and 2).

\section{Comparison of patients with definite EB and PA with those judged to be indeterminate}

When comparing the 67 patients with definite EB with the 172 patients with EB judged to be indeterminate, patients with

\begin{tabular}{|c|c|c|c|}
\hline \multirow[t]{2}{*}{ TABLE 3} & \multicolumn{3}{|c|}{$\begin{array}{l}\text { Overview of incidences of definite and } \\
\text { indeterminate Enterobacteriaceae (EB)/ } \\
\text { Pseudomonas aeruginosa (PA) according to } \\
\text { different denominators }\end{array}$} \\
\hline & Subjects $n$ & $\begin{array}{c}\text { EB in total } \\
\text { population }{ }^{\#} \%\end{array}$ & $\begin{array}{l}\text { EB in population with } \\
\text { respiratory samples } \%\end{array}$ \\
\hline Definite $\mathrm{EB}^{+}$ & 67 & 1.3 & 3.2 \\
\hline Indeterminate E & 172 & 3.4 & 8.2 \\
\hline Definite $\mathrm{PA}^{+}$ & 22 & 1.0 & 0.4 \\
\hline Indeterminate $\mathrm{P}$ & 27 & 0.5 & 1.3 \\
\hline
\end{tabular}

definite EB were significantly older, were more frequently nursing home residents, had more comorbid conditions, showed greater pneumonia severity on presentation (CRB-65, hospitalisation rate and requirement for mechanical ventilation) and had a higher mortality (table 1). This was similar in terms of nursing home residency, comorbidity and mortality when comparing patients with PA as definite pathogens to the respective group judged to be indeterminate. However, due to small sample sizes, differences were nonsignificant except hospitalisation rate (table 2).

\section{Predictive factors of CAP due to EB/PA}

The following factors were predictors of CAP due to EB: age $>65$ yrs, nursing home residency, enteral tube feeding, congestive heart failure, renal failure, cerebrovascular disease, other chronic neurological disorders, and diabetes. On multivariate analysis, congestive heart failure and cerebrovascular disease were predictive of EB (table 4).

Predictors of PA on univariate analysis included nursing home residency, enteral tube feeding, chronic respiratory disease, cerebrovascular disease, and other chronic neurological disorders. Independent predictors of CAP due to PA included chronic respiratory disease and enteral tube feeding (table 5).

\section{Antimicrobial treatment of CAP due to EB/PA}

Based on the available susceptibility testing, first-line antimicrobial therapy in patients with EB in respiratory samples 


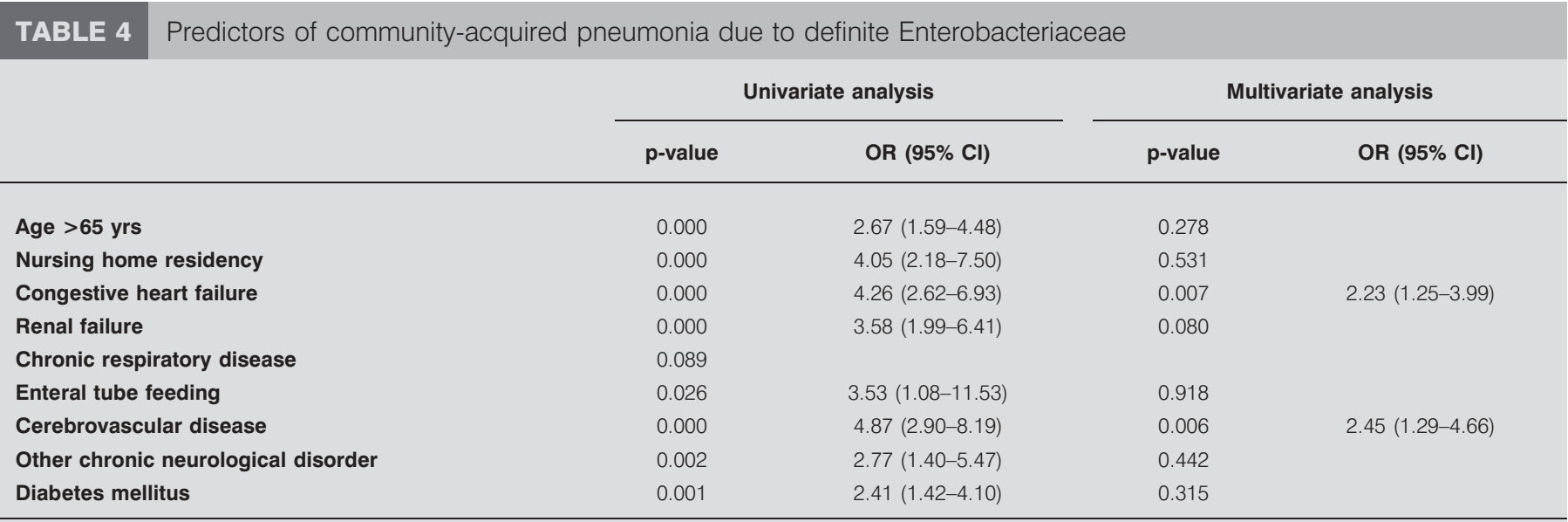

OR: odds ratio; $\mathrm{Cl}$ : confidence interval.

and/or blood cultures was adequate in 17 (25\%) patients, inadequate in $15(22 \%)$ and indeterminate in 35 (53\%) (tables 1 and 2 of online supplementary material).

Likewise, in patients with definite PA pneumonia, initial antimicrobial treatment was inadequate in $14(64 \%)$ out of 22 cases due to innate resistance of the pathogen to the chosen antimicrobial agents. Four (18\%) patients received adequate antibiotic therapy according to susceptibility testing, and, in the remaining four (18\%) cases, adequacy remained indeterminate (table 3 of online supplementary material).

\section{Outcome of CAP due to EB/PA}

The 30-day mortality of CAP due to EB/PA was substantially higher than in patients without respiratory samples available and without these pathogens. The mortality of CAP with EB as definite pathogens in respiratory samples was $20 \%$, EB bacteraemia $22 \%$ and PA pneumonia 18\% (tables 1 and 2).

\section{Predictors of mortality}

Amongst others, the presence of either EB or PA as definite pathogen, but not as indeterminate, was identified as a risk factor on univariate analysis. However, only EB, and not PA, remained an independent predictor on multivariate analysis (table 6).

\section{DISCUSSION}

In earlier studies, including a general population of hospitalised patients with CAP, only few showed a proportion of $>3 \%$ of CAP due to EB $[16,17]$, the highest reaching 9\% [18]. Likewise, in a recent study addressing this particular issue, the incidence was 3\% [2]. The incidence of EB in severe CAP was up to $15 \%$, which is 2-3-fold higher than in the general hospitalised population $[19,20]$. However, in a large study of patients aged $\geqslant 65 \mathrm{yrs}$, the incidence was 9.6\% [21]. Moreover, in a study addressing the impact of HCAP, the incidence was $11.6 \%$ in CAP and even reached $19.7 \%$ in HCAP patients [10]. In the present study, the overall incidence was $1.3 \%$. It should be noted that the incidence was minimal in ambulatory patients.

The reasons for this wide range of observed incidences are complex. First, a very strict definition was used, relying on not only quality criteria for sputum samples but also semiquantitative and quantitative cultures. Indeed, four out of five

TABLE 5 Predictors of community-acquired pneumonia due to definite Pseudomonas aeruginosa

\begin{tabular}{|c|c|c|c|c|}
\hline & \multicolumn{2}{|c|}{ Univariate analysis } & \multicolumn{2}{|c|}{ Multivariate analysis } \\
\hline & p-value & OR $(95 \% \mathrm{Cl})$ & p-value & OR $(95 \% \mathrm{Cl})$ \\
\hline Age $>65$ yrs & 0.374 & & & \\
\hline Nursing home residency & 0.001 & $4.94(1.81-13.50)$ & 0.885 & \\
\hline Congestive heart failure & 0.461 & & & \\
\hline Renal failure & 0.678 & & & \\
\hline Chronic respiratory disease & 0.010 & $4.01(1.63-9.86)$ & 0.001 & $4.78(1.89-12.07)$ \\
\hline Enteral tube feeding & 0.000 & $21.79(7.80-60.90)$ & 0.000 & $13.87(3.39-56.65)$ \\
\hline Cerebrovascular disease & 0.000 & $4.64(1.88-11.44)$ & 0.107 & \\
\hline Other chronic neurological disorder & 0.040 & $3.49(1.17-10.39)$ & 0.698 & \\
\hline Diabetes mellitus & 0.108 & & & \\
\hline
\end{tabular}

OR: odds ratio; $\mathrm{Cl}$ : confidence interval. 
TABLE 6 Predictors of 30-day mortality on univariate and multivariate analysis

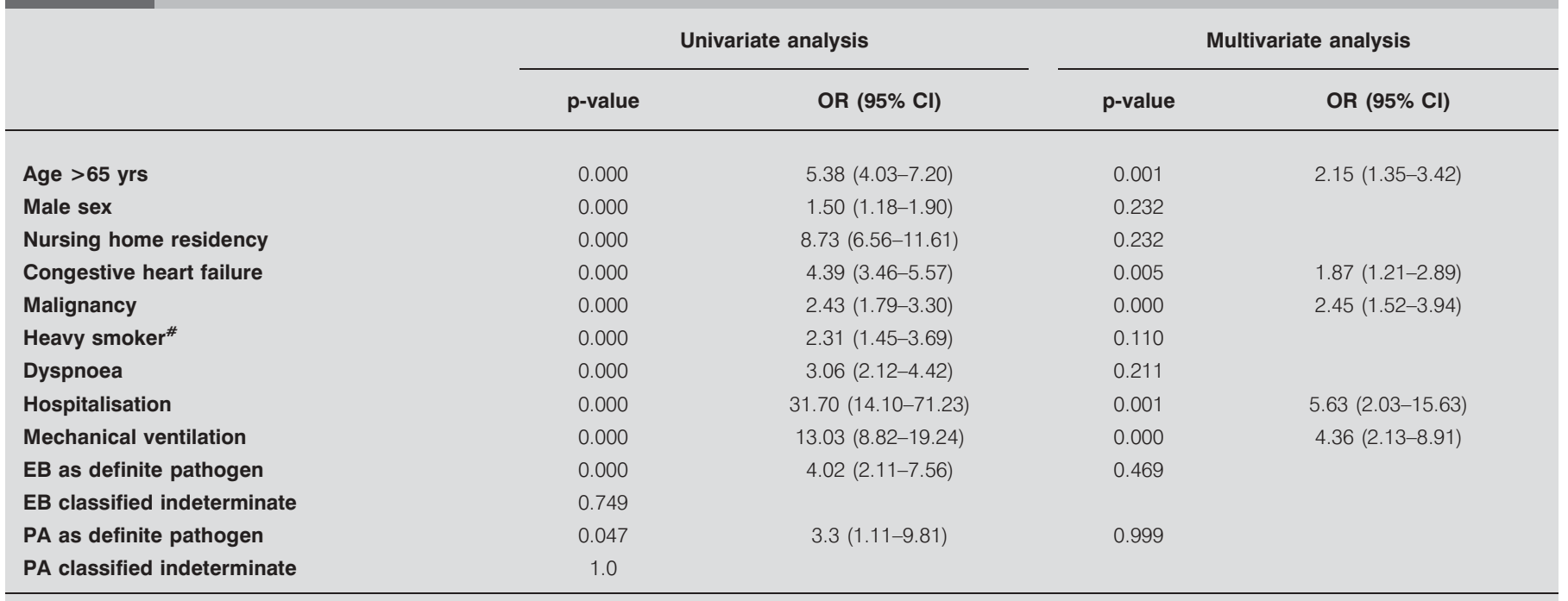

OR: odds ratio; Cl: confidence interval; EB: Enterobacteriaceae; PA: Pseudomonas aeruginosa. ${ }^{\#}:>20$ pack-yrs.

EB isolates were judged to represent an indeterminate status. Although it cannot be ruled out that the present case definition may have missed a few additional patients with CAP due to $\mathrm{EB}$, the finding of striking differences as regards pneumonia severity on presentation and clinical outcome in the groups with definite EB compared to the group judged to be indeterminate are in support of the case definition. Secondly, the incidence of EB seems to be highly dependent upon the population studied. It was found that CAP due to EB was associated with greater age, a broad range of comorbid conditions and more severe pneumonia on admission. Thus, since the proportion of patients with severe pneumonia was limited, a higher rate might be expected in studies including a higher proportion of patients with severe CAP.

Although the incidence of CAP due to EB was low, mortality was very high, reaching $20 \%$ in definite cases diagnosed from a respiratory sample and $22 \%$ in bacteraemic cases. This rate was three to five times higher than that in the general study population. It should be noted that this finding does not necessarily imply excess mortality from these pathogens, and may simply indicate an elderly and more severely disabled population. Indeed, every fifth patient with CAP due to definite EB resided in a nursing home, and comorbidity was extensive. The mortality rate exceeded the rate of mechanical ventilation (21 versus 13\%), indicating that treatment restrictions due to prognostic considerations were applied. Although the yield of blood cultures in CAP is generally low, and their routine use has, therefore, been questioned [22, 23], it was found that a large proportion of CAP due to EB presents with bacteraemia. Therefore, we argue that blood cultures should be obtained, especially in patients at risk of EB.

The patients typically at risk of CAP due to EB can be characterised quite confidently. They are elderly, highly comorbid and highly acutely disabled patients. Surprisingly, chronic respiratory disease could not be confirmed as a risk factor $[2,20]$. However, comorbid conditions, such as cardiac and cerebral disease, could be identified as independent predictors. Unfortunately, no data were available regarding antimicrobial pretreatment and hospitalisation in the preceding 3-6 months, nor were there any data regarding aspiration at our disposal, factors which have been implicated in CAP due to EB in a previous study [2].

Many findings regarding CAP due to EB can also be applied to CAP due to PA. Again, the reported incidence varied considerably in previous studies. Of 10 recent studies including $>250$ patients, six did not find any cases due to PA $[6,7,16$, $17,24,25]$, and the incidence in the remaining four was only $1-5 \%[4,21,26,27]$. Likewise, in severe CAP, the incidence was higher, reaching $12 \%$ [19]. In a previous Spanish study, PA was the third most common pathogen, with an incidence of $7 \%$ [2]. Interestingly, this finding could not be reproduced in the most recent study from this group, where the incidence of PA reached only 2 and 3\% in non-intensive care unit (ICU) and ICU patients, respectively [28]. Consistent with previous findings, PA was absent in ambulatory patients [29]. In hospitalised patients also, only very few cases due to PA were found. Similarly to EB, most (55\%) isolates of PA were considered to be indeterminate, an assumption once again confirmed by dramatic differences in mortality. Whereas CAP due to definite PA exhibited a mortality of $18 \%$, mortality was $6 \%$ in the general population, and only $4 \%$ in the population judged to be indeterminate with regard to PA.

Only very few cases of CAP due to PA have been described in previously healthy patients [30]. Accordingly, only one patient without pre-existing comorbid conditions was found in the present population. In hospitalised patients, it appears that the clinical characteristics of patients with CAP due to PA are similar to those of EB. However, the clinical predictors were not identical; CAP due to PA was associated with chronic respiratory disease and enteral tube feeding, the latter being the most powerful predictor. As in CAP due to $E B$, the mortality rate exceeded the rate of mechanical ventilation (18 
versus 9\%). Again, this does not necessarily imply excess mortality due to PA.

The present findings do not support the concept of HCAP to be generally applicable in Germany and countries with a comparable epidemiological situation. First, the incidence of unusual pathogens, such as methicillin-resistant S. aureus, EB or PA, was very low in the present study, although $6 \%$ of the population consisted of nursing home residents. This might be due to significant differences in the characteristics of nursing home residents and infection control procedures when comparing German and US nursing homes [31]. Secondly, resistance rates in EB were low in the present study, with only one E. coli isolate identified as an extended-spectrum $\beta$ lactamase-positive microorganism. Likewise, none of the patients in the present study with PA had a multidrugresistant isolate.

The present study has some limitations. First, respiratory samples and blood cultures were not available for all of the patients studied. Indeed, the group without respiratory samples was older, more comorbid, and showed greater pneumonia severity and higher mortality. Thus some cases with EB/PA may have been missed in this group. However, the mortality was still substantially lower than that in the definite EB/PA group (7.3 versus $20.9 \%$ for EB and $18.2 \%$ for $\mathrm{PA}$ ), and was similar to the indeterminate groups (7\% for EB; $3.7 \%$ for PA). Secondly, the number of patients with severe CAP was limited. Since pneumonia severity is a risk factor for $\mathrm{CAP}$ due to $\mathrm{EB} / \mathrm{PA}$, the incidence of both may have been underestimated in severe cases. However, it appears improbable that patients presenting with severe CAP exhibit risk factors substantially different to those described in the present study. Therefore, the data are still of help in identifying those patients at risk. Finally, standardised susceptibility testing was not available for the majority of patients with EB, limiting the analysis regarding inappropriate treatment.

In conclusion, strict diagnostic criteria have to be applied in order to avoid overestimation of EA and PA. We argue that the present data provide sufficient support for the view that these pathogens show a low incidence and are mainly observed in patients at defined risk. A risk-adapted approach of initial antimicrobial treatment and consistent microbial investigation in patients at increased risk and not assigned to treatment restrictions remains mandatory. At least in Germany and countries with a comparable epidemiological situation, such an approach probably permits better guidance of patient management than the concept of HCAP.

\section{SUPPORT STATEMENT}

The Competence Network for Community-Acquired Pneumonia is supported by the German Ministry of Education and Research (Berlin, Germany).

\section{STATEMENT OF INTEREST}

None declared.

\section{ACKNOWLEDGEMENTS}

The German Competence Network for Community-Acquired Pneumonia (CAPNETZ) is a multidisciplinary approach to the better understanding and treatment of patients with community-acquired pneumonia. The network has only been made possible by the contribution of many investigators. We are especially indebted to the work of the investigators in the local clinical centres who established and maintained contact with all practitioners, physicians and respiratory specialists cooperating within the network.

In addition, we would like to acknowledge the work of the central computing unit and the central service unit, with A. Sawazki (Institute for Medical Microbiology and Hygiene, Ulm University Hospital, Ulm, Germany) providing excellent technical support.

It is also our responsibility and pleasure to express our appreciation to all of the clinical physicians and physicians in private practice who saw and identified patients with community-acquired pneumonia for their work dedicated to CAPNETZ.

\section{REFERENCES}

1 Ewig S. Community-acquired pneumonia: definition, epidemiology, and outcome. Semin Respir Infect 1999; 14: 94-102.

2 Arancibia F, Bauer TT, Ewig S, et al. Community-acquired pneumonia due to Gram-negative bacteria and Pseudomonas aeruginosa: incidence, risk, and prognosis. Arch Intern Med 2002; 162: 1849-1858.

3 Kang CI, Song JH, Oh WS, et al. Clinical outcomes and risk factors of community-acquired pneumonia caused by Gram-negative bacilli. Eur J Clin Microbiol Infect Dis 2008; 27: 657-661.

4 Blanquer J, Blanquer R, Borras R, et al. Aetiology of community acquired pneumonia in Valencia, Spain: a multicentre prospective study. Thorax 1991; 46: 508-511.

5 Miyashita N, Shimizu H, Ouchi K, et al. Assessment of the usefulness of sputum Gram stain and culture for diagnosis of community-acquired pneumonia requiring hospitalization. Med Sci Monit 2008; 14: CR171-CR176.

6 Charles PG, Whitby M, Fuller AJ, et al. The etiology of communityacquired pneumonia in Australia: why penicillin plus doxycycline or a macrolide is the most appropriate therapy. Clin Infect Dis 2008; 46: 1513-1521.

7 Lieberman D, Schlaeffer F, Boldur I, et al. Multiple pathogens in adult patients admitted with community-acquired pneumonia: a one year prospective study of 346 consecutive patients. Thorax 1996; 51: 179-184.

8 Kollef MH, Shorr A, Tabak YP, et al. Epidemiology and outcomes of health-care-associated pneumonia: results from a large US database of culture-positive pneumonia. Chest 2005; 128: 3854-3862.

9 Guay DR. Guidelines for the management of adults with health care-associated pneumonia: implications for nursing facility residents. Consult Pharm 2006; 21: 719-725.

10 Micek ST, Kollef KE, Reichley RM, et al. Health care-associated pneumonia and community-acquired pneumonia: a single-center experience. Antimicrob Agents Chemother 2007; 51: 3568-3573.

11 Shorr AF, Zilberberg MD, Micek ST, et al. Prediction of infection due to antibiotic-resistant bacteria by select risk factors for health careassociated pneumonia. Arch Intern Med 2008; 168: 2205-2210.

12 Shindo Y, Sato S, Maruyama E, et al. Health-care-associated pneumonia among hospitalized patients in a Japanese community hospital. Chest 2009; 135: 633-640.

13 Welte T, Suttorp N, Marre R. CAPNETZ - community-acquired pneumonia competence network. Infection 2004; 32: 234-238.

14 Mauch H, Lütticken R, Gatermann S, eds. MiQ 7. Infektionen der tiefen Atemwege. Teil I. Qualitätsstandards in der mikrobiologischinfektiologischen Diagnostik. [Infection of the Deep Airways. Part I. Quality Standards in the Microbiological Diagnosis of Infectious Diseases.] Jena, Gustav-Fischer-Verlag, 1999.

15 Mauch H, Lütticken R, Gatermann S, eds. MiQ 8. Infektionen der tiefen Atemwege. Teil II. Qualitätsstandards in der mikrobiologischinfektiologischen Diagnostik. [Infection of the Deep Airways. Part II. Quality Standards in the Microbiological Diagnosis of Infectious Diseases.] Jena, Gustav-Fischer-Verlag, 1999. 
16 Fang GD, Fine M, Orloff J, et al. New and emerging etiologies for community-acquired pneumonia with implications for therapy. A prospective multicenter study of 359 cases. Medicine (Baltimore) 1990; 69: 307-316.

17 Neill AM, Martin IR, Weir R, et al. Community acquired pneumonia: aetiology and usefulness of severity criteria on admission. Thorax 1996; 51: 1010-1016.

18 Lim I, Shaw DR, Stanley DP, et al. A prospective hospital study of the aetiology of community-acquired pneumonia. Med J Aust 1989; 151: 87-91.

19 Ewig S, Torres A. Severe community-acquired pneumonia. Clin Chest Med 1999; 20: 575-587.

20 Rello J, Rodriguez A, Torres A, et al. Implications of COPD in patients admitted to the intensive care unit by communityacquired pneumonia. Eur Respir J 2006; 27: 1210-1216.

21 Kaplan V, Angus DC, Griffin MF, et al. Hospitalized communityacquired pneumonia in the elderly: age- and sex-related patterns of care and outcome in the United States. Am J Respir Crit Care Med 2002; 165: 766-772.

22 Campbell SG, Marrie TJ, Anstey R, et al. Utility of blood cultures in the management of adults with community acquired pneumonia discharged from the emergency department. Emerg Med 2003; 20: 521-523.

23 Campbell SG, Marrie TJ, Anstey R, et al. The contribution of blood cultures to the clinical management of adult patients admitted to the hospital with community-acquired pneumonia: a prospective observational study. Chest 2003; 123: 1142-1150.
24 Howard LS, Sillis M, Pasteur MC, et al. Microbiological profile of community-acquired pneumonia in adults over the last 20 years. J Infect 2005; 50: 107-113.

25 Van der Eerden MM, Vlaspolder F, de Graaff CS, et al. Comparison between pathogen directed antibiotic treatment and empirical broad spectrum antibiotic treatment in patients with communityacquired pneumonia: a prospective randomised study. Thorax 2005; 60: 672-678.

26 Ortiz-Ruiz G, Vetter N, Isaacs R, et al. Ertapenem versus ceftriaxone for the treatment of community-acquired pneumonia in adults: combined analysis of two multicentre randomized, double-blind studies. J Antimicrob Chemother 2004; 53: Suppl. 2, ii59-ii66.

27 Ruiz M, Ewig S, Marcos MA, et al. Etiology of communityacquired pneumonia: impact of age, comorbidity, and severity. Am J Respir Crit Care Med 1999; 160: 397-405.

28 Liapikou A, Ferrer M, Polverino E, et al. Severe communityacquired pneumonia: validation of the Infectious Diseases Society of America/American Thoracic Society guidelines to predict an intensive care unit admission. Clin Infect Dis 2009; 48: 377-385.

29 Marrie TJ, Poulin-Costello M, Beecroft MD, et al. Etiology of community-acquired pneumonia treated in an ambulatory setting. Respir Med 2005; 99: 60-65.

30 Hatchette TF, Gupta R, Marrie TJ. Pseudomonas aeruginosa community-acquired pneumonia in previously healthy adults: case report and review of the literature. Clin Infect Dis 2000;31: 1349-1356.

31 Wendt $\mathrm{C}$, Bock-Hensley $\mathrm{O}$, von Baum H. Infection control in German nursing homes. Am J Infect Control 2006; 34: 426-429. 